\title{
Dissociating viewpoint costs in mental rotation and object recognition
}

\author{
WILLIAM G. HAYWARD \\ University of Hong Kong, Hong Kong, China \\ GUOMEI ZHOU \\ Chinese University of Hong Kong, Hong Kong, China \\ and Chinese Academy of Sciences, Beijing, China \\ ISABEL GAUTHIER \\ Vanderbilt University, Nashville, Tennessee \\ and \\ IRINA M. HARRIS \\ University of Sydney, Sydney, Australia
}

\begin{abstract}
In a mental rotation task, participants must determine whether two stimuli match when one undergoes a rotation in 3-D space relative to the other. The key evidence for mental rotation is the finding of a linear increase in response times as objects are rotated farther apart. This signature increase in response times is also found in recognition of rotated objects, which has led many theorists to postulate mental rotation as a key transformational procedure in object recognition. We compared mental rotation and object recognition in tasks that used the same stimuli and presentation conditions and found that, whereas mental rotation costs increased relatively linearly with rotation, object recognition costs increased only over small rotations. Taken in conjunction with a recent brain imaging study, this dissociation in behavioral performance suggests that object recognition is based on matching of image features rather than on 3-D mental transformations.
\end{abstract}

Starting from the early 1970 s, Shepard and colleagues (Shepard \& Cooper, 1982; Shepard \& Judd, 1976; Shepard \& Metzler, 1971) conducted a series of experiments showing that mental transformations of 2-D shapes and 3-D objects appeared to follow the same laws as physical transformations of real objects. This finding of mental rotation was a cornerstone in the foundations of the emerging field of cognitive science, since it showed that the physical world constrains internal mental representations. The typical mental rotation task is one in which participants are asked to determine whether two stimuli are identical or mirror reflections (i.e., differing in handedness); the difficulty lies in the fact that the stimuli are rotated relative to each other. The hallmark of mental rotation is that response times form a linear function of orientation difference between the two stimuli.

Whereas mental rotation tasks require a discrimination between mirror images of the same object across view-

This article was supported by a grant from the Research Grants Council of the Hong Kong Special Administrative Region, China (project number HKU 4260/03H) to W.G.H. We thank Michael Corballis, Robert Nosofsky, and two anonymous reviewers for their comments on the manuscript, and Olivia Cheung, Hoi-Yan Lam, and Keung-Tat Lee for their assistance in carrying out the research project. Correspondence relating to this article may be sent to W. G. Hayward, Department of Psychology, University of Hong Kong, Pokfulam Road, Hong Kong (e-mail: whayward@hku.hk). points, studies of object recognition often involve discriminations among different objects across viewpoints. Many object recognition studies have found results similar to those of mental rotation studies, with recognition judgments becoming slower (thus, more difficult) as an object is rotated away from a studied viewpoint (e.g., Hayward \& Williams, 2000; Jolicœur, 1985; Tarr \& Pinker, 1989; Tarr, Williams, Hayward, \& Gauthier, 1998). Mental rotation has often been invoked to account for these effects (see, e.g., Jolicœur, 1990; Tarr \& Pinker, 1989). According to these theories, objects are represented at specific viewpoints; when encountered from a novel view, the perceived stimulus is mentally rotated until it matches a stored view, at which point the recognition decision can be made.

In recent years, however, a number of studies have contested the view that rotated object recognition relies on mental rotation, both on theoretical grounds (e.g., Corballis, 1988) and from empirical evidence. For example, Lawson and Jolicœur (2003) showed that viewpoint costs for objects rotated in the picture plane contained nonlinearities that cannot be accommodated by mental rotation. Willems and Wagemans (2001) tested recognition of misoriented objects rotated around a number of different axes; they found no effect of axis on performance, even though an explicit mental rotation task showed large differences. Jolicœur, Corballis, and Lawson (1998) rotated objects in the picture plane and had participants either identify the 
object (object recognition) or say whether it would face left or right in an upright orientation (mental rotation). They found that perceived or actual rotation of the object affected mental rotation but not object recognition, again casting doubt on a common mechanism for both tasks. De Caro and Reeves (2000) examined naming and orientation judgments for misoriented objects that were backward masked; orientation judgments showed roughly linear viewpoint costs, but naming judgments showed costs that asymptoted past $60^{\circ}$ rotations. In addition, indirect measures of object recognition, such as repetition blindness and priming, suggest that the initial activation of familiar object representations is independent of orientation and, implicitly, of mental rotation (Harris \& Dux, 2005a, 2005b). Finally, neuropsychological studies have revealed a double dissociation between recognizing rotated objects and performing mental rotation (Harris, Harris, \& Caine, 2002; Turnbull \& McCarthy, 1996).

In these studies, with the exception of Willems and Wagemans (2001), objects were familiar and rotations occurred in the picture plane. ${ }^{1}$ Taken collectively, the studies show strong evidence that recognition of familiar objects misoriented in the picture plane cannot be explained by mental rotation. However, the original studies of mental rotation and its application to object recognition were studied in a rather different situation. Shepard and colleagues used objects that consisted of sets of identical cubes connected in different ways; as such, the objects lacked qualitatively distinct features and required recognition that was based on overall structure. Many studies of object recognition cited as support for mental rotation accounts (e.g., Rock \& DiVita, 1987; Tarr, 1995; Tarr \& Pinker, 1989) have used similar objects. Given that many theorists have proposed qualitatively distinct processes for object recognition at different levels of categorical specificity (e.g., bird vs. sparrow; Biederman, 1987; Farah, 1990; Tarr \& Bülthoff, 1995), the possibility remains that mental rotation might underlie recognition of objects that are qualitatively similar and lack distinct identifying features.

In a previous study on this topic (Gauthier et al., 2002), we used objects similar to those of Shepard and Metzler (1971) and compared mental rotation and object recognition, using fMRI to measure cortical activation. Both tasks required sequential matching of two novel stimuli; in mental rotation, the stimuli always depicted the same object but differed in handedness; in object recognition, the stimuli could be the same object or two different objects. If mental rotation is the basis for recognition of these objects, we would expect to find similar brain regions underlying performance in the two tasks. In fact, we found distinct regions associated with each task. Performance in the mental rotation task was associated with activity in the superior parietal lobe within the dorsal extrastriate visual pathway, which is held to be responsible for spatial processing (Ungerleider \& Mishkin, 1982) and response programming (Milner \& Goodale, 1995). In contrast, object recognition recruited occipito-temporal areas within the ventral visual pathway; activity in these regions seems related to object shape (Logothetis \& Pauls, 1995; Tanaka, 1996; Vogels, Biederman, Bar, \& Lorincz, 2001).

Given this neuroanatomical distinction, we propose different causes for viewpoint costs in mental rotation and object recognition. According to this theoretical account, mental rotation produces viewpoint costs because of the additional time taken to internally rotate the object through a larger rotation, as in 3-D space; therefore, performance costs in this task should be predicted directly from the extent of the rotation necessary to bring the two views into alignment. On the other hand, viewpoint costs in object recognition are attributed to the relative differences in the appearance of the object as seen in two different views. In this situation, viewpoint costs would be tied only indirectly to the size of the actual object rotation; in many cases, there may be a correlation between size of rotation and image similarity, but such a correspondence need not hold across all views.

Tentative evidence for this proposal comes from Logothetis and Pauls (1995), who recorded activity of cells in the inferior temporal lobe of primates. They found strong view specificity in many cells, so that activity was strongest for a particular view and gradually reduced to baseline across rotations in depth of about $30^{\circ}$. However, a strong response was also found in many cases as views approached $180^{\circ}$ rotations of the objects, presumably because the appearance of the object was more similar to a mirror-image reflection of the original view (see also Perrett, Oram, \& Ashbridge, 1998; Rollenhagen \& Olson, 2000). On this basis, object recognition might be expected to be quicker and easier with rotations in depth approaching $180^{\circ}$ than at intermediate viewpoints, whereas mental rotation should be most difficult at $180^{\circ}$ because this involves the longest possible rotation back to the studied view.

In the present study, we examined differences in viewpoint costs for mental rotation and object recognition using qualitatively similar objects. From the results of Shepard and colleagues, we expected mental rotation to show consistent linear costs of viewpoint change. In contrast, if object recognition has a cortical basis in the ventral visual pathway (Gauthier et al., 2002; Logothetis \& Pauls, 1995), we expected object recognition performance to degrade across small rotations but then to improve at rotations approaching $180^{\circ}$.

One concern with experiments such as these is that recognition performance might be affected by the specific views tested. For example, one would always expect best performance when the stimulus is unchanged - that is, at the $0^{\circ}$ viewpoint. Equally, we were concerned that testing $180^{\circ}$ might lead to artificially good performance because, for objects with minimal self-occlusion (such as ours), the $180^{\circ}$ viewpoint represents an approximation of a simple mirror-image reflection. Therefore, testing a range of views including $0^{\circ}$ and $180^{\circ}$ might produce results in the form of a quadratic function, because of good performance with the two views at the ends of the continuum. To control for this problem, we tested views between $15^{\circ}$ and $165^{\circ}$ inclusively, rather than between $0^{\circ}$ and $180^{\circ}$. 


\section{METHOD}

\section{Participants}

Twenty-four undergraduates from the Chinese University of Hong Kong participated in the study for either payment or course credit. Six participants were excluded from the analysis; 5 because performance in at least one of the tasks was at chance, and 1 because of a failure in understanding the task. Thus, data from 18 participants were included in the analysis.

\section{Materials}

Four 3-D objects, originally used in the Gauthier et al. (2002) study, were rendered from seven viewpoints: To create our $0^{\circ}$ figures, we arbitrarily started with a view in which all components of the object were oriented left-right or front-back with respect to the viewer, and then rotated the view $10^{\circ}$ to create some perspective depth cues; the other six views were obtained by rotating the object in depth in $30^{\circ}$ steps around a vertical axis running through its vertically oriented central component, starting with $15^{\circ}$ (and thus ending with $165^{\circ}$ ). Mirror images were created for every view. Figure 1 shows all objects in the $0^{\circ}$ view, along with all seven views for one object. The images were about $8.4 \mathrm{~cm}$ high and $5-13 \mathrm{~cm}$ wide. Viewing position was not fixed.

\section{Design}

Two independent variables were manipulated within subjects. First, the participants performed either a mental rotation task or an object recognition task. Second, the two views of each object were separated by $15^{\circ}, 45^{\circ}, 75^{\circ}, 105^{\circ}, 135^{\circ}$, or $165^{\circ}$.

\section{Procedure}

The experiment was conducted using RSVP software (www tarrlab.org) on an eMac with a 17-in. CRT monitor. Each participant performed one block of mental rotation trials and one block of object recognition trials, with each task using two of the objects; the identity of the objects in each task and the order of blocks were counterbalanced across participants. In the mental rotation task, the participants were informed that on every trial they would see two identical objects rotated relative to each other, and that the objects might be the same or different in terms of handedness. The participants were asked to decide as quickly and accurately as possible whether the two views showed the object with the same or different handedness, while ignoring the difference caused by viewpoint. For object recognition, each trial showed either the same object twice or two different objects. Again, the stimuli could differ in handedness and always differed in viewpoint; the participants were instructed to ignore handedness and viewpoint and to judge whether the two stimuli showed the same or different objects. Before each block, the participants received instructions and 10 practice trials, during
(A)

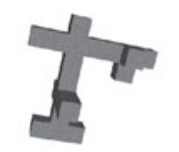

Object 1

(B)

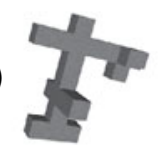

$15^{\circ}$

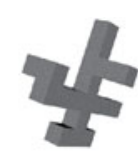

Object 2

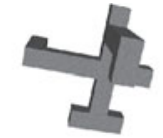

Object 3

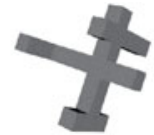

Object 4
Figure 1. (A) The four objects used in the experiment. (B) The six possible rotated views of Object 1. which they were free to ask questions of the experimenter, followed by 480 test trials. Each trial consisted of a 500-msec fixation cross, followed by the first stimulus for $500 \mathrm{msec}$, and then the second stimulus for $3,000 \mathrm{msec}$ or until the participant made a response. The first stimulus was always at the $0^{\circ}$ viewpoint; the second stimulus was at one of the other six viewpoints. Responses were made by pressing one of two keys on the keyboard. Feedback was given in the form of an audible beep following incorrect responses.

\section{RESULTS}

Both RTs and accuracy were recorded. For data analysis, trials were discarded if RTs were more than three standard deviations from the mean (1.57\% of trials). To control for possible response biases, sensitivity was computed from hits and false alarms using a logistic distribution to calculate $d_{\mathrm{L}}$ (Snodgrass \& Corwin, 1988). Like $d^{\prime}$, higher $d_{\mathrm{L}}$ scores represent a larger difference between hits and false alarms and are, therefore, indicative of better performance. ${ }^{2}$ Means for both RTs from correct trials and $d_{\mathrm{L}}$ were computed for individual participants, and these means were subjected to a $2 \times 6$ within-subjects ANOVA with task (mental rotation or object recognition) and viewpoint difference $\left(15^{\circ}, 45^{\circ}, 75^{\circ}, 105^{\circ}, 135^{\circ}\right.$, or $\left.165^{\circ}\right)$ as the independent variables.

\section{Response Times}

The mean RTs to correct same trials are shown in Figure 2. The ANOVA showed a significant main effect of task, with responses in the mental rotation task slower than in the object recognition task $[F(1,17)=10.35, p<$ $.01]$. Responses also varied across differences in viewpoint $[F(5,85)=27.42, p<.001]$. However, these effects were modulated by a significant interaction between viewpoint and task $[F(5,85)=13.82, p<.001]$ : Whereas responses in mental rotation showed a fairly consistent slowing with increases in viewpoint change, responses in object recognition did not show this pattern, but rather flattened out past a rotation of $45^{\circ}$, with a reversal of the function toward $165^{\circ}$.

This interpretation is supported by linear and quadratic contrasts that were performed separately for mental rotation and object recognition. In mental rotation, the linear component was significant $[F(1,17)=86.1, p<.001]$, but the quadratic component was not $[F(1,17)=1.52$, $p>$.1]. Object recognition, however, also showed a significant linear trend $[F(1,17)=8.42, p<.05]$, but with a significant quadratic component as well $[F(1,17)=16.37$, $p<.01]$. Therefore, for mental rotation, RT increased linearly as a function of angle of rotation, whereas for object recognition, the increases in RT followed a more quadratic function with respect to viewpoint.

\section{Sensitivity}

Performance as assessed by $d_{\mathrm{L}}$ is displayed in Figure 3 . Using the same type of ANOVA performed on RTs, main effects were found for both task $[F(1,17)=86.12, p<$ $.001]$ and viewpoint $[F(5,85)=24.88, p<.001]$. The interaction was marginally significant $[F(5,85)=2.19$, 


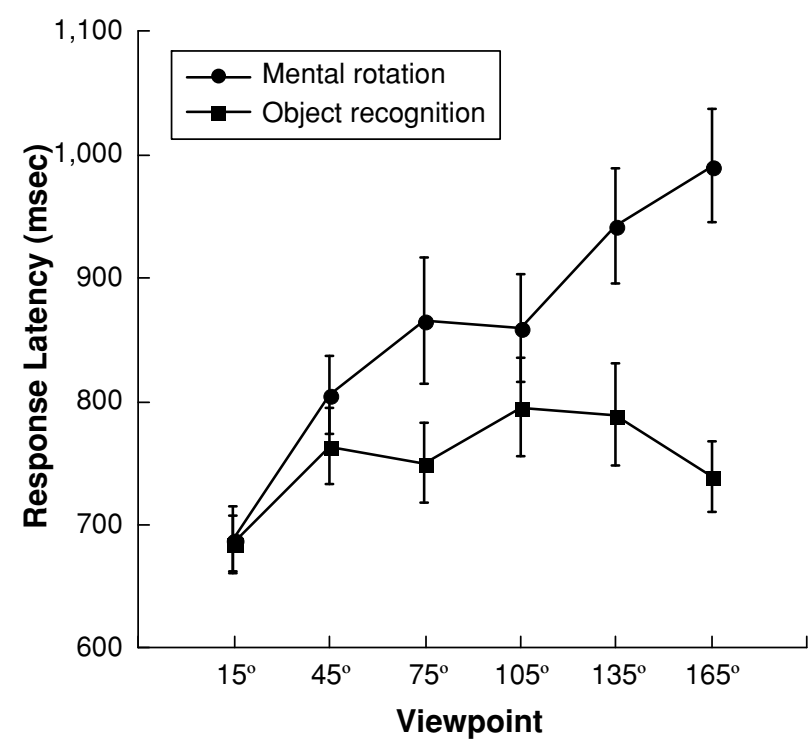

Figure 2. Response latencies for the experiment. Here and elsewhere, error bars show standard errors of the mean.

$p=.063]$, which was consistent with the finding of larger viewpoint costs for mental rotation than for object recognition with RTs, but here the results were somewhat weaker. Since the main effect of viewpoint appeared to occur between $15^{\circ}$ and $45^{\circ}$, we computed separate one-way ANOVAs to examine viewpoint costs for mental rotation and object recognition between $45^{\circ}$ and $165^{\circ}$. No differences were observed within this range of viewpoints in either mental rotation $[F(4,68)=1.57, p=.19]$ or object recognition $(F<1)$. Therefore, the effect of viewpoint in the original omnibus ANOVA seems to be due to differences between the $15^{\circ}$ and $45^{\circ}$ rotations; larger rotations did not cause additional impairments in sensitivity in either task.

\section{DISCUSSION}

The results of this study show that with identical stimuli and with tasks that are identical apart from the requirement to attend to the handedness or identity of the objects, patterns of viewpoint costs in mental rotation and object recognition are surprisingly different. Whereas mental rotation took systematically longer with increasing viewpoint difference between the two stimuli on a trial, latencies in object recognition showed an inverted U-shaped pattern, with performance getting slower as the size of the viewpoint difference between two stimuli increased to about $90^{\circ}$, but getting faster once again as the viewpoint difference continued to increase toward $180^{\circ}$. Although sensitivity was better for object recognition than for mental rotation, and though for object recognition it did not show the quadratic function observed in the latencies, there was no evidence that modulations of performance in latencies were achieved at the cost of sensitivity. This overall pattern of results is consistent with physiological studies that have shown that neurons in the inferior temporal lobe prefer specific views of objects but are also activated by large rotations of that view (e.g., Logothetis \& Pauls, 1995; Perrett et al., 1998).

Of course, these conclusions depend on our tasks' abilities to measure mental rotation and object recognition. Although the former ability seems relatively uncontroversial, since our task was essentially the same one devised by Shepard and Metzler (1971), our recognition task distinguished between only two objects that had no qualitatively distinct features, and thus may have suffered from problems of generalizability. Reducing the stimulus set to its smallest possible size (two objects) probably meant that participants became very familiar with specific features and configurations. On the other hand, viewpoint costs were almost certainly increased by the lack of qualitative features that distinguished the objects (Biederman, 1987; Hummel, 2001).

Although particular aspects of the experimental paradigm may have affected the costs of viewpoint change, we are confident that the strategies employed are appropriate for our theoretical goal. First, Tarr et al. (1998), among other studies, showed that large variations in experimental techniques have relatively small effects on performance in object recognition studies; for example, the use of sequential matching paradigms such as the one used here showed results qualitatively similar to those of longer term, name learning tasks. Second, our goal was to compare mental rotation and object recognition in the context tested by Shepard and colleagues. Many researchers (e.g., Corballis, Zbrodoff, Shetzer, \& Butler, 1978; Jolicœur, 1985; Jolicœur \& Milliken, 1989) have reported a similar finding with recognition of familiar objects rotated in the picture plane - that is, naming latencies with roughly linear costs up to rotations of about $120^{\circ}$, but an improvement of performance at $180^{\circ}$. The theoretical rationale of the present study was not to mimic recognition of these familiar and qualitatively distinct objects; in fact, we have good reason to believe that rotations in depth with such objects

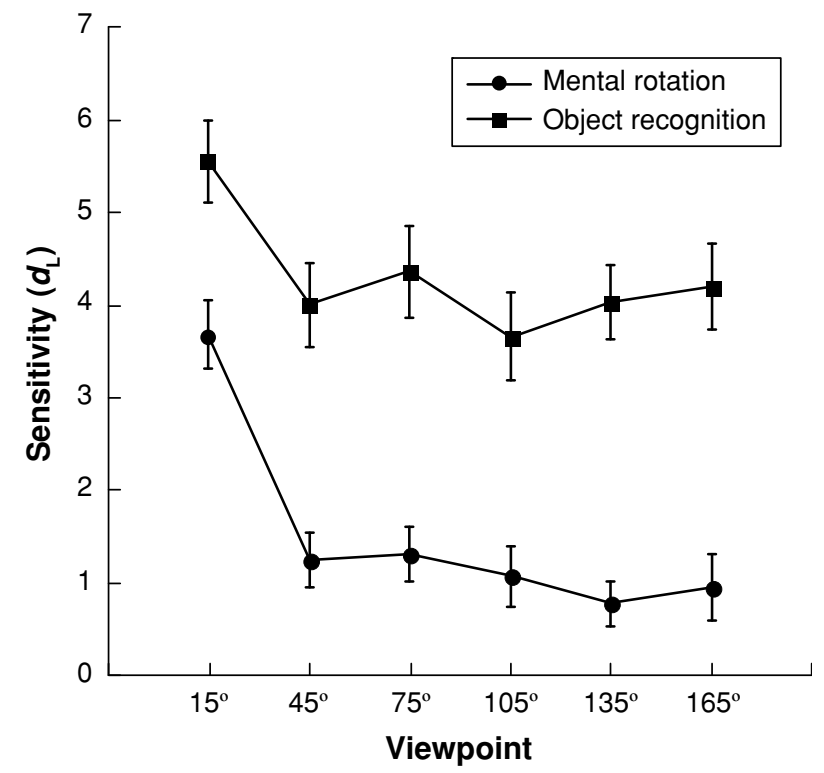

Figure 3. Sensitivity for the experiment, plotted using $d_{L}$. 
would show even flatter U-shaped functions than those observed here (Palmer, Rosch, \& Chase, 1981). Rather, we have been able to show with a task similar to that used by Shepard that recognition costs are not consistent with those of mental rotation; this seems strong evidence that mental rotation and object recognition employ distinct processes, suggesting that performance costs in object recognition are caused by some type of comparison of object features. Thus, although across small rotations performance costs are positively related to viewpoint, across larger rotations this relationship changes to a negative one as increases in viewpoint difference produce a corresponding increase in the similarity of image features.

In our view, differences in behavioral performance for mental rotation and object recognition have their basis in the distinction between the processing characteristics of the dorsal and ventral extrastriate visual pathways. Parietal lobe activity mediates visual control of our physical interaction with the environment, and therefore could be viewed as the embodiment of Shepard's (1981) hypothesis that internal representations are functionally isomorphic with the external objects to which they refer. If activity in the parietal lobe supports compensation for a change in viewpoint - as is the case for mental rotation - costs in terms of the time taken to complete the task would be expected to show the same effects as completing the task in the physical world (that is, as actually rotating the object). Therefore, a close correspondence would be expected between the size of the physical rotation and the time taken to complete the task.

Activity in the ventral visual stream, on the other hand, appears to mediate quite different visual representations. Neurons in areas within this stream, such as the lateraloccipital complex and the inferior temporal lobe, are sensitive to visual features of stimuli (Logothetis \& Pauls, 1995; Logothetis \& Sheinberg, 1996; Tanaka, 1996). A number of theorists (e.g., Milner \& Goodale, 1995; Vogels et al., 2001) have proposed that ventral-stream activity supports perceptual representations of objects as part of a wider network that allows for activation of conscious, semantic representations of the world around us, for the purposes of categorization and identification. Behaviors under the control of the ventral stream are therefore less likely to show a correlation with 3-D transformations (such as rotation) and might instead be based more directly on some aspect of image correspondence between the stimuli.

The results of this article contribute to the evidence that mental rotation and object recognition are distinct cognitive processes, even though in some instances they may show relatively similar patterns of results. This is not to suggest that there is no overlap between these processes; clearly they will share much physiological processing in primary visual cortex, and they may also share neural networks in much higher regions of both the dorsal and ventral visual pathways. However, they have clear distinctions, which can be seen here behaviorally, and elsewhere in fMRI (Gauthier et al., 2002) and in neuropsychological dissociations (Harris et al., 2002). Even for visually similar objects lacking in distinctive features, mental rotation seems not to explain viewpoint costs in object recognition.

\section{REFERENCES}

BiEderman, I. (1987). Recognition-by-components: A theory of human image understanding. Psychological Review, 94, 115-147.

Corballis, M. C. (1988). Recognition of disoriented shapes. Psychological Review, 95, 115-123.

Corballis, M. C., Zbrodoff, N. J., Shetzer, L. I., \& Butler, P. B. (1978). Decisions about identity and orientation of rotated letters and digits. Memory \& Cognition, 6, 98-107.

De Caro, S. A., \& ReEves, A. (2000). Rotating objects to determine orientation, not identity: Evidence from a backward-masking/dual-task procedure. Perception \& Psychophysics, 62, 1356-1366.

FARAH, M. J. (1990). Visual agnosia: Disorders of object recognition and what they tell us about normal vision. Cambridge, MA: MIT Press, Bradford Books.

Gauthier, I., Hayward, W. G., Tarr, M. J., Anderson, A. W., SkudLARSKI, P., \& GoRE, J. C. (2002). BOLD activity during mental rotation and viewpoint-dependent object recognition. Neuron, 34, 161-171.

Harris, I. M., \& DuX, P. E. (2005a). Orientation-invariant object recognition: Evidence from repetition blindness. Cognition, 95, 73-93.

Harris, I. M., \& DuX, P. E. (2005b). Orientation-invariant representations are activated first in object recognition [Abstract]. Perception, 34(Suppl.), 128.

Harris, I. M., HARris, J. A., \& CAine, D. (2002). Mental rotation deficits following damage to the right basal ganglia. Neuropsychology, 16, 524-537.

Hayward, W. G., \& Williams, P. (2000). Viewpoint dependence and object discriminability. Psychological Science, 11, 7-12.

Hummel, J. E. (2001). Complementary solutions to the binding problem in vision: Implications for shape perception and object recognition. Visual Cognition, 8, 489-517.

Jolicceur, P. (1985). The time to name disoriented natural objects. Memory \& Cognition, 13, 289-303.

JoliCGeUR, P. (1990). Identification of disoriented objects: A dual-systems theory. Mind \& Language, 5, 387-410.

Joliceur, P., Corballis, M. C., \& Lawson, R. (1998). The influence of perceived rotary motion on the recognition of rotated objects. Psychonomic Bulletin \& Review, 5, 140-146.

Jolicceur, P., \& Milliken, B. (1989). Identification of disoriented objects: Effects of context of prior presentation. Journal of Experimental Psychology: Learning, Memory, \& Cognition, 15, 200-210.

LAwson, R., \& JoliceEUR, P. (2003). Recognition thresholds for planerotated pictures of familiar objects. Acta Psychologica, 112, 17-41.

Logothetis, N. K., \& Pauls, J. (1995). Psychophysical and physiological evidence for viewer-centered object representations in the primate. Cerebral Cortex, 5, 270-288.

Logothetis, N. K., \& Sheinberg, D. L. (1996). Visual object recognition. Annual Review of Neuroscience, 19, 577-621.

Milner, A. D., \& Goodale, M. A. (1995). The visual brain in action. Oxford: Oxford University Press.

Palmer, S., Rosch, E., \& Chase, P. (1981). Canonical perspective and the perception of objects. In J. Long \& A. Baddeley (Eds.), Attention and performance $I X$ (pp. 135-151). Hillsdale, NJ: Erlbaum.

Perrett, D. I., Oram, M. W., \& Ashbridge, E. (1998). Evidence accumulation in cell populations responsive to faces: An account of generalisation of recognition without mental transformations. Cognition, 67, 111-145.

Rock, I., \& DiViTA, J. (1987). A case of viewer-centered object perception. Cognitive Psychology, 19, 280-293.

Rollenhagen, J. E., \& Olson, C. R. (2000). Mirror-image confusion in single neurons of the macaque inferotemporal cortex. Science, 287, 1506-1508.

ShePARD, R. N. (1981). Psychophysical complementarity. In M. Kubovy \& J. R. Pomerantz (Eds.), Perceptual organization (pp. 297-341). Hillsdale, NJ: Erlbaum.

Shepard, R. N., \& Cooper, L. A. (1982). Mental images and their transformations. Cambridge, MA: MIT Press. 
SHEPARD, R. N., \& JUdD, S. A. (1976). Perceptual illusion of rotation of three-dimensional objects. Science, 191, 952-954.

Shepard, R. N., \& Metzler, J. (1971). Mental rotation of threedimensional objects. Science, 171, 701-703.

SNodgrass, J. G., \& Corwin, J. (1988). Pragmatics of measuring recognition memory: Applications to dementia and amnesia. Journal of Experimental Psychology: General, 117, 34-50.

TANaKa, K. (1996). Inferotemporal cortex and object vision. Annual Review of Neuroscience, 19, 109-139.

TARR, M. J. (1995). Rotating objects to recognize them: A case study on the role of viewpoint dependency in the recognition of threedimensional objects. Psychonomic Bulletin \& Review, 2, 55-82.

TARR, M. J., \& BÜLTHOFF, H. H. (1995). Is human object recognition better described by geon structural descriptions or by multiple views? Comment on Biederman and Gerhardstein (1993). Journal of Experimental Psychology: Human Perception \& Performance, 21, 1494-1505.

TARR, M. J., \& PINKER, S. (1989). Mental rotation and orientationdependence in shape recognition. Cognitive Psychology, 21, 233-282.

Tarr, M. J., Williams, P., Hayward, W. G., \& Gauthier, I. (1998). Three-dimensional object recognition is viewpoint dependent. Nature Neuroscience, 1, 275-277.

Turnbull, O. H., \& McCarthy, R. A. (1996). When is a view unusual?
A single case study of orientation-dependent visual agnosia. Brain Research Bulletin, 40, 497-503.

UngerLeIDER, L. G., \& MishKin, M. (1982). Two cortical visual systems. In D. J. Ingle, M. A. Goodale, \& R. J. W. Mansfield (Eds.), Analysis of visual behavior (pp. 549-586). Cambridge, MA: MIT Press

Vogels, R., Biederman, I., BAR, M., \& LoRincZ, A. (2001). Inferior temporal neurons show greater sensitivity to nonaccidental than to metric shape differences. Journal of Cognitive Neuroscience, 13, 444-453.

Willems, B., \& Wagemans, J. (2001). Matching multicomponent objects from different viewpoints: Mental rotation as normalization? Journal of Experimental Psychology: Human Perception \& Performance, 27, 1090-1115.

\section{NOTES}

1. Willems and Wagemans (2001) used unfamiliar but qualitatively distinct objects, as well as a variety of axes for rotation.

2. $d_{\mathrm{L}}$ is calculated using the formula $d_{\mathrm{L}}=\ln \{[\mathrm{H}(1-\mathrm{FA})] /[(1-\mathrm{H}) \mathrm{FA}]\}$, where $\mathrm{H}$ and FA represent hits and false alarms, respectively.

(Manuscript received August 9, 2005; revision accepted for publication March 4, 2006.) 